\title{
ALK Folic Acid Transport and Integrin Signal Induced-Angiogenesis Network in Human Hepatocellular Carcinoma (HCC) by Systems- Theoretical Analysis
}

\author{
Juxiang Huang, Lin Wang*, Minghu Jiang and Hong Lin \\ Biomedical Center, School of Electronics Engineering, Beijing University of Posts and Telecommunications, Beijing, 100876, China
}

\begin{abstract}
We constructed the high-expression ALK activated transport and signal network in human hepatocellular carcinoma (HCC) compared with low-expression (fold change $\geq 2$ ) no-tumor hepatitis/cirrhotic tissues (HBV or HCV infection) in GEO data set, by using integration of gene regulatory activated and inhibited network inference method with gene ontology (GO) analysis. Our result showed that ALK transport and signal upstream network ECT2, FOLR1, GNAZ, GRM1, ITGA2, LEF1, NR5A1, PTHLH, RIMS3, SORT1, SOX2 activated ALK, and downstream ALK-activated BAP1, CAD, CDH13, CNTNAP2, GRM1, ITGA2, LAPTM4B, MAP2K6, NR5A1, STMN1 in HCC. We obtained that the different biological processes of ALK activated network consisted of folic acid transport, cell surface receptor linked signal transduction, cell-cell signaling, G-protein coupled receptor protein signaling pathway, integrin-mediated signaling pathway, intracellular signaling cascade, low density lipoprotein mediated signaling, Rac protein signal transduction, Rho protein signal transduction in HCC compared with the activated network of no-tumor hepatitis/cirrhotic tissues, as a result of inducing folic acid transport and integrin signal induced-angiogenesis in HCC. Our hypothesis was verified by the different and the same biological processes of ALK activated transport and signal network of HCC compared with the corresponding inhibited network of no-tumor hepatitis/cirrhotic tissues and HCC, respectively.
\end{abstract}

Keywords: ALK; Human hepatocellular carcinoma (HCC); Folic acid transport and integrin signal induced-angiogenesis network; Systems-theoretical analysis

\section{Introduction}

ALK is our identified significant high-expression gene in human hepatocellular carcinoma (HCC) compared with low-expression (fold change $\geq 2$ ) no-tumor hepatitis/cirrhotic tissues (HBV or HCV infection) from GEO data set GSE10140-10141 (http://www.ncbi.nlm. nih.gov/geo/query/acc.cgi?acc=GSE10140, http://www.ncbi.nlm.nih. gov/geo/query/acc.cgi?acc=GSE10141). ALK is related to integral to plasma membrane, membrane; nucleotide binding, transmembrane receptor protein tyrosine kinase activity, receptor signaling protein tyrosine kinase activity, receptor activity, protein binding, ATP binding, transferase activity; protein amino acid phosphorylation, protein amino acid N-linked glycosylation, transmembrane receptor protein tyrosine kinase signaling pathway, nervous system development, brain development (GO database).

Folic acid transport, integrin signal and lipoprotein are involved in angiogenesis. Such as, Folic-acid-mediated inhibition of human colon-cancer cell growth [1]; The Foxc2 transcription factor modulates angiogenesis via induction of integrin beta 3 expression [2]; alphavbeta3 integrin and a moody integrin angiogenesis in a changing environment [3]; Active tumor targeting of nonmaterial's using folic acid, integrin receptors and transferring [4]; Role of tetraspanin CD151-alpha3/alpha6 integrin complex in angiogenesis [5]; Integrin affinity modulation in angiogenesis [6]; RECK function of ss1-integrin-dependent in physiologic and tumor angiogenesis [7]; Activation of Ras/MAP kinase is required in high density lipoprotein-induced angiogenesis in human coronary artery endothelial cells [8]; Collateral formation Impairment in lipoprotein(a) transgenic mice therapeutic angiogenesis induced by human hepatocyte growth factor gene [9]; Homocysteine and folic acid effects on angiogenesis and VEGF expression during chicken vascular development [10]; Pharmacological inhibition of integrin alphavbeta3 aggravates experimental liver fibrosis and inhibits hepatic angiogenesis [11]; Lipoprotein contributes to angiogenesis on the chick embryo chorioallantoic membrane [12]; Interaction of alpha9beta1 integrin with thrombospondin-1 promotes angiogenesis [13]; Reconstituted high-density lipoprotein enhances differentiation of endothelial progenitor cells and stimulates ischemia-induced angiogenesis [14]; Angiogenesis requires Beta1 integrin expression on endothelial cells but not vasculogenesis [15]; Integrin alpha9beta1 directly binds to vascular endothelial growth factor (VEGF)-A and induces VEGF-A-induced angiogenesis [16]; A novel mediator of ovarian angiogenesis follicular fluid high density lipoprotein-associated sphingosine 1-phosphate [17]; Integrin-linked kinase modulates melanoma angiogenesis by activating NF-kappaB/interleukin-6 signaling pathway [18]; Relationship between oxidized lipoprotein, human coronary atherosclerotic plaque stabilization and angiogenesis [19]; Possible roles for folic acid in the modulation of trophoblast invasion and placental development in normal early human pregnancy [20]; Alpha(5)beta(1) integrin ligand PHSRN contributes invasion and alpha(5) mRNA in endothelial cells to stimulate angiogenesis [21]. Yet the distinct high-expression ALK folic acid transport and integrin signal induced-angiogenesis network in HCC remains to be elucidated. Here we constructed the highexpression ALK activated transport and signal network in HCC from GEO data set by gene regulatory network inference method based on linear programming and decomposition procedure.

In this study, we constructed ALK up- and down-stream activated and inhibited transport and signal network in no-tumor hepatitis/ cirrhotic tissues and HCC. The biological process and data analysis of

*Corresponding author: Lin Wang, Biomedical Center, School of Electronics Engineering, Beijing University of Posts and Telecommunications, Beijing, 100876 China, Tel: 0086-13240981826; Fax: 8610-62785736 (Minghu Jiang c/o Lin Wang); E-mail: wanglin98@tsinghua.org.cn

Received November 08, 2011; Accepted February 23, 2012; Published February 25,2012

Citation: Huang J, Wang L, Jiang M, Lin H (2012) ALK Folic Acid Transport and Integrin Signal Induced-Angiogenesis Network in Human Hepatocellular Carcinoma (HCC) by Systems-Theoretical Analysis. Mol Biol 1:102. doi:10.4172/21689547.1000102

Copyright: $\odot 2012$ Huang J, et al. This is an open-access article distributed under the terms of the Creative Commons Attribution License, which permits unrestricted use, distribution, and reproduction in any medium, provided the original author and source are credited. 
the low- and high-expression ALK transport and signal network was done in no-tumor hepatitis/cirrhotic tissues (HBV or HCV infection) and HCC by GO database. By comparison with the same and different gene ontology (GO) of ALK activated and inhibited transport and signal network between no-tumor hepatitis/cirrhotic tissues and HCC, we put forwards hypothesis of ALK activated transport and signal network of inducing folic acid transport and integrin signal inducedangiogenesis in HCC.

\section{Materials and Methods}

We used microarrays containing 6,144 genes from 25 no-tumor hepatitis/cirrhotic tissues and 25 HCC patients in GEO data set GSE10140-10141(http://www.ncbi.nlm.nih.gov/geo/query/acc. cgi?acc=GSE10140, http://www.ncbi.nlm.nih.gov/geo/query/acc. cgi?acc $=$ GSE10141). The raw microarray data was preprocessed by log base 2 .

Significant expressed genes of ALK transport and signal network were identified using significant analysis of microarrays (SAM) (http:// wwwstat.stanford.edu/ tibs/SAM/) [22]. We selected two classes unpaired and minimum fold change $\geq 2$ and chose the significant highly expressed value genes of HCC compared with that of no-tumor hepatitis/cirrhotic tissues (HBV or HCV infection) under the falsediscovery rate and q-value were $0 \%$. The q-value is like the well-known $\mathrm{P}$-value, but adapted to multiple-testing situations.

ALK transport and signal network was constructed based on GRNInfer and GVedit tools (http://www.graphviz.org/About. php). GRNInfer is a novel mathematic method called GNR (Gene Network Reconstruction tool) based on linear programming and a decomposition procedure for inferring gene network [23]. We established ALK activated network of HCC based on the fold change $\geq 2$ distinguished genes and selected parameters as lambda 0.0 because we used one data set. Lambda was a positive parameter which balanced the matching and sparsity terms in the objective function. Using different thresholds, we could predict various networks with the different edge density. The threshold parameters make the edge whose strength of link is smaller than threshold not shown in the network graph. The smaller this parameter, the more edges in the network graph. We selected threshold 1.0e-7.

ALK transport and signal network of HCC was analyzed using Molecule Annotation System, MAS (CapitalBio Corporation, Beijing, China; http://bioinfo.capitalbio.com/mas3/). MAS is a Web-based software toolkit for a whole data mining and function annotation solution to extract and analyze biological molecules relationships from public databases. The primary databases of MAS integrated various well-known biological resources, such as Gene Ontology (http://www. geneontology.org), KEGG (http://www.genome.jp/kegg/), BioCarta (http://www.biocarta.com/), GenMapp (http://www.genmapp.org/), HPRD (http://www.hprd.org/), MINT (http://mint.bio.uniroma2.it/ mint/Welcome.do), BIND (http://www.blueprint.org/), Intact (http:// www.ebi.ac.uk/intact/), UniGene (www.ncbi.nlm.nih.gov/UniGen), OMIM (http://www.ncbi.nlm.nih. gov/entrez/query.fcgi?db=OMIM) and disease (http://bioinfo.capitalbio.com/mas3/). MAS offers various query entries and graphics. The algorithm is $\mathrm{P}, \mathrm{Q}$ value in GO and pathway of module was presented in reference [24].

\section{Results}

We constructed ALK up- and down-stream activated and inhibited transport and signal network in no-tumor hepatitis/cirrhotic tissues and HCC from our total network of 225 significant high-expression molecules (fold change $\geq 2$ ) from 6,144 genes of 25 HCC compared with 25 no-tumor hepatitis/cirrhotic tissues (HBV or HCV infection) by GRNInfer, respectively.

We extracted the biological process of GO terms and did numbers data analysis of the different biological processes of ALK activated transport and signal network in HCC compared with activated network of no-tumor hepatitis/cirrhotic tissues, the same biological processes of ALK activated transport and signal network in HCC compared with

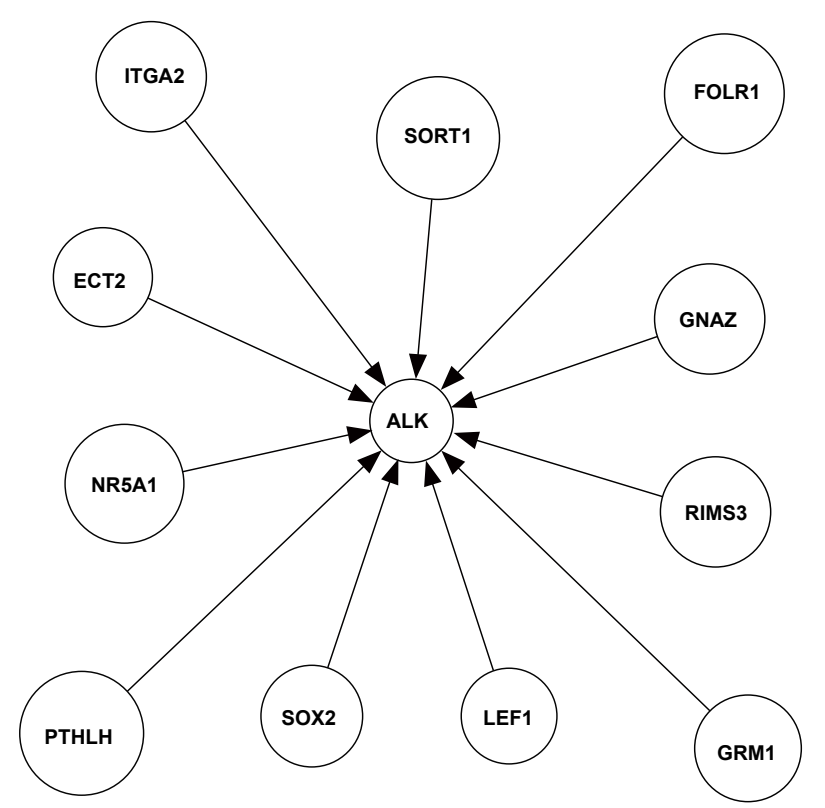

Figure 1: ALK upstream activated transport and signal network in HCC by GRNInfer. Arrowhead represents activation relationship.

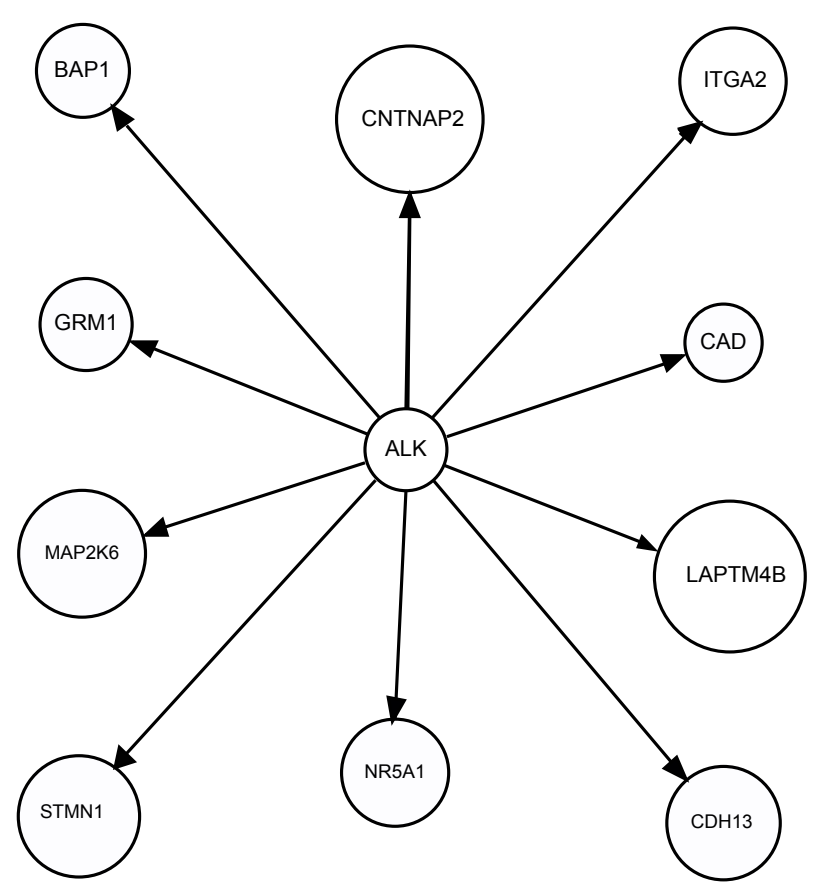

Figure 2: ALK downstream activated transport and signal network in HCC by GRNInfer. Arrowhead represents activation relationship. 
Citation: Huang J, Wang L, Jiang M, Lin H (2012) ALK Folic Acid Transport and Integrin Signal Induced-Angiogenesis Network in Human Hepatocellular Carcinoma (HCC) by Systems-Theoretical Analysis. Mol Biol 1:102. doi:10.4172/2168-9547.1000102

Page 3 of 5

\begin{tabular}{|l|c|l|l|l|}
\hline \multicolumn{5}{|c|}{ The Different ALK Activated Transport and Signal Network of HCC compared with Activated Network of No-tumor } \\
Hepatitis/cirrhotic Tissues
\end{tabular}

Table 1: GO Terms and numbers data analysis of the different biological processes of ALK activated transport and signal network of HCC compared with activated network of no-tumor hepatitis/cirrhotic tissues, the same biological processes of ALK activated transport and signal network of HCC compared with inhibited network of no-tumor hepatitis/cirrhotic tissues.

\begin{tabular}{|l|c|l|}
\hline \multicolumn{1}{|c|}{ The Different ALK Activated Transport and Signal Network of HCC compared with Inhibited Network of HCC } \\
\hline \multicolumn{1}{|c|}{ Terms } & Numbers & \multicolumn{1}{|c|}{ Nerms } \\
\hline endosome to lysosome transport & 1 & intracellular signaling cascade \\
\hline folic acid transport & 1 & integrin-mediated signaling pathway \\
\hline Golgi to endosome transport & $\mathbf{1}$ & Rac protein signal transduction \\
\hline plasma membrane to endosome transport & $\mathbf{1}$ & Rho protein signal transduction \\
\hline endosome transport via multivesicular body sorting pathway & $\mathbf{1}$ & low density lipoprotein mediated signaling \\
\hline cell surface receptor linked signal transduction & $\mathbf{1}$ & induction of apoptosis by extracellular signals \\
\hline
\end{tabular}

Table 2: GO Terms and numbers data analysis of the different biological processes of ALK activated transport and signal network of HCC compared with inhibited network of HCC.

inhibited network of no-tumor hepatitis/cirrhotic tissues, as shown in Table 1. GO terms and numbers data was analyzed the different biological processes of ALK activated compared with inhibited transport and signal network in HCC, as shown in Table 2.

ALK activated transport and signal network was constructed in HCC. Our result showed that upstream ECT2, FOLR1, GNAZ, GRM1, ITGA2, LEF1, NR5A1, PTHLH, RIMS3, SORT1, SOX2 activated ALK, and the downstream ALK-activated BAP1, CAD, CDH13, CNTNAP2, GRM1, ITGA2, LAPTM4B, MAP2K6, NR5A1, STMN1 in HCC, as shown in Figure 1 and Figure 2.

\section{Discussion}

Our aim is to construct, interpret, verify and predict the function of novel high-expression ALK folic acid transport and integrin signal induced-angiogenesis network in HCC. We have already constructed and analyzed some novel molecular network from different databases presented in our articles [24-37]. In this study, we constructed ALK up- and down-stream activated and inhibited transport and signal network in no-tumor hepatitis/cirrhotic tissues and HCC. The biological process and data analysis of the low- and high-expression ALK transport and signal network was done in no-tumor hepatitis/ cirrhotic tissues (HBV or HCV infection) and HCC by GO database. By comparison with the same and different gene ontology (GO) of ALK activated and inhibited transport and signal network between notumor hepatitis/cirrhotic tissues and HCC, we put forwards hypothesis of ALK activated transport and signal network of inducing folic acid transport and integrin signal induced-angiogenesis in HCC.

We extracted the biological process of GO terms and did numbers data analysis of the different biological processes of ALK activated transport and signal network in HCC compared with activated network of no-tumor hepatitis/cirrhotic tissues, the same biological processes of ALK activated transport and signal network in HCC compared with inhibited network of no-tumor hepatitis/cirrhotic tissues (Table
1 and Table 2). We constructed the high-expression ALK activated transport and signal network in human hepatocellular carcinoma (HCC) compared with low-expression (fold change $\geq 2$ ) no-tumor hepatitis/cirrhotic tissues (HBV or HCV infection) in GEO data set using integration of gene regulatory network inference method. Our result showed that ALK transport and signal upstream network ECT2, FOLR1, GNAZ, GRM1, ITGA2, LEF1, NR5A1, PTHLH, RIMS3, SORT1, SOX2 activated ALK, and downstream ALK-activated BAP1, CAD, CDH13, CNTNAP2, GRM1, ITGA2, LAPTM4B, MAP2K6, NR5A1, STMN1 (Figure 1 and Figure 2) in HCC.

By further comparison with the same and different gene ontology (GO) of ALK activated and inhibited transport and signal network between no-tumor hepatitis/cirrhotic tissues and HCC, we obtained that the different biological processes of ALK activated network consisted of folic acid transport, cell surface receptor linked signal transduction, cell-cell signaling, G-protein coupled receptor protein signaling pathway, integrin-mediated signaling pathway, intracellular signaling cascade, low density lipoprotein mediated signaling, Rac protein signal transduction, Rho protein signal transduction in HCC compared with activated network of no-tumor hepatitis/cirrhotic tissues, as a result of inducing folic acid transport and integrin signal induced-angiogenesis in HCC.

The same biological processes of ALK activated network included transport, cell surface receptor linked signal transduction, cell-cell signaling, G-protein signaling, signal transduction in HCC compared with inhibited network of no-tumor hepatitis/cirrhotic tissues. It is consistent with the different biological processes of ALK activated transport and signal network of HCC compared with activated network of no-tumor hepatitis/cirrhotic tissues.

The different biological processes of ALK activated network consisted of endosome to lysosome transport, endosome transport via multivesicular body sorting pathway, folic acid transport, Golgi to 
endosome transport, plasma membrane to endosome transport, cell surface receptor linked signal transduction, induction of apoptosis by extracellular signals, integrin-mediated signaling pathway, intracellular signaling cascade, low density lipoprotein mediated signaling, Rac protein signal transduction, Rho protein signal transduction in HCC compared with inhibited network of HCC. It is consistent with the different biological processes of ALK activated transport and signal network of HCC compared with activated network of notumor hepatitis/cirrhotic tissues, the same biological processes of ALK activated transport and signal network of HCC compared with inhibited network of no-tumor hepatitis/cirrhotic tissues, respectively.

Therefore, our ALK activated folic acid transport and integrin signal induced-angiogenesis hypothesis was verified by the different and the same biological processes of ALK activated transport and signal network of HCC compared with the corresponding inhibited network of no-tumor hepatitis/cirrhotic tissues and HCC, respectively.

\section{Acknowledgments}

This work was supported by the National Natural Science Foundation of China (No.60871100) and (No.61171114), the Returned Overseas Chinese Scholars for Scientific research Foundation of State Education Ministry, Significant Science and Technology Project for New Transgenic Biological Species (2009ZX08012001B), Automatical Scientific Planning of Tsinghua University (20111081023 and 20111081010), State Key Lab of Pattern Recognition Open Foundation.

\section{References}

1. Duthie SJ (2001) Folic-acid-mediated inhibition of human colon-cancer cell growth. Nutrition 17: 736-737.

2. Hayashi, H, Sano H, Seo S, Kume T (2008) The Foxc2 transcription factor regulates angiogenesis via induction of integrin beta3 expression. J Biol Chem 283: 23791-23800.

3. Hodivala-Dilke K (2008) alphavbeta3 integrin and angiogenesis: a moody integrin in a changing environment. Curr Opin Cell Biol 20: 514-519.

4. Kolhatkar R, Lote A, Khambati $\mathrm{H}$ (2011) Active tumor targeting of nanomaterials using folic acid, transferrin and integrin receptors. Curr Drug Discov Technol 8 : 197-206.

5. Liu, W F, Zuo HJ, Chai BL, Peng D, Fei YJ, et al. (2011) Role of tetraspanin CD151- $\alpha 3 / \alpha 6$ integrin complex: Implication in angiogenesis CD151-integrin complex in angiogenesis. Int J Biochem Cell Biol 43: 642-650.

6. Mahabeleshwar GH, Chen J, Feng W, Somanath PR, Razorenova, et al. (2008) Integrin affinity modulation in angiogenesis. Cell Cycle 7: 335-347.

7. Miki T, Shamma A, Kitajima S, Takegami Y, Noda M, et al. (2010) The $\beta 1$ integrin-dependent function of RECK in physiologic and tumor angiogenesis. Mol Cancer Res 8: 665-676.

8. Miura S, Fujino M, Mastsuo Y, Kawamura A, Tanigawa H, et al. (2003) High density lipoprotein-induced angiogenesis requires the activation of Ras/MAP kinase in human coronary artery endothelial cells. Arterioscler Thromb Vasc Biol 23: 802-808.

9. Morishita R, Sakaki M, Yamamoto K, Iguchi S, Aoki M, et al. (2002) Impairment of collateral formation in lipoprotein(a) transgenic mice: therapeutic angiogenesis induced by human hepatocyte growth factor gene. Circulation 105: 1491-1496.

10. Oosterbaan AM, Steegers EA, Ursem NT (2012) The effects of homocysteine and folic acid on angiogenesis and VEGF expression during chicken vascular development. Microvasc Res 83: 98-104.

11. Patsenker E, Popov Y, Stickel F, Schneider V, Ledermann M, et al. (2009) Pharmacological inhibition of integrin alphavbeta3 aggravates experimental liver fibrosis and suppresses hepatic angiogenesis. Hepatology 50: 1501-1511.

12. Ribatti D, Vacca A, Giachetta F, Cesaretti S, Anichini M, et al. (1998) Lipoprotein (a) induces angiogenesis on the chick embryo chorioallantoic membrane. Eur J Clin Invest 28: 533-537.

13. Staniszewska I, Zaveri S, Del Valle L, Oliva I, Rothman VL, et al. (2007) Interaction of alpha9beta1 integrin with thrombospondin-1 promotes angiogenesis. Circ Res 100: 1308-1316.

14. Sumi M, Sata M, Miura S, Rye KA, Toya N, et al. (2007) Reconstituted highdensity lipoprotein stimulates differentiation of endothelial progenitor cells and enhances ischemia-induced angiogenesis. Arterioscler Thromb Vasc Biol 27 813-818.

15. Tanjore H, Zeisberg EM, Gerami-Naini B, Kalluri R (2008) Beta1 integrin expression on endothelial cells is required for angiogenesis but not for vasculogenesis. Dev Dyn 237: 75-82.

16. Vlahakis NE, Young BA, Atakilit A, Hawkridge AE, Issaka RB, et al. (2007) Integrin alpha9beta1 directly binds to vascular endothelial growth factor (VEGF)-A and contributes to VEGF-A-induced angiogenesis. J Biol Chem 282 $15187-15196$.

17. von Otte S, Paletta JR, Becker S, Konig S, Fobker M, et al. (2006) Follicular fluid high density lipoprotein-associated sphingosine 1-phosphate is a nove mediator of ovarian angiogenesis. J Biol Chem 281: 5398-5405.

18. Wani AA, Jafarnejad SM, Zhou J, Li G (2011) Integrin-linked kinase regulates melanoma angiogenesis by activating NF-kB/interleukin- 6 signaling pathway Oncogene 30: 2778-2788.

19. Wi, LX, Tang QH, Sun L, Shi HY, Guo AT, et al. (2006) [Relationship between oxidized lipoprotein, angiogenesis and human coronary atherosclerotic plaque stabilization]. Zhonghua Bing Li Xue Za Zhi 35: 138-141.

20. Williams PJ, Bulmer JN, Innes BA, Broughton Pipkin F (2011) Possible roles for folic acid in the regulation of trophoblast invasion and placental development in normal early human pregnancy. Biol Reprod 84: 1148-1153.

21. Zeng ZZ, Yao H, Staszewski ED, Rockwood KF, Markwart SM, et al. (2009) alpha(5)beta(1) Integrin Ligand PHSRN Induces Invasion and alpha(5) mRNA in Endothelial Cells to Stimulate Angiogenesis. Transl Oncol 2: 8-20.

22. Storey JD (2002) A direct approach to false discovery rates. J Roy Stat Soc Ser B 64: 479-498

23. Wang $Y$, Joshi $T$, Zhang XS, Ku D, Chen L, et al. (2006) Inferring gene regulatory networks from multiple microarray datasets. Bioinformatics 22: 2413-2420.

24. Wang L, Sun L, Huang J, Jiang M (2011) Cyclin-dependent kinase inhibito 3 (CDKN3) novel cell cycle computational network between human nonmalignancy associated hepatitis/cirrhosis and hepatocellular carcinoma (HCC) transformation. Cell Prolif 44: 291-299.

25. Huang J, Wang L, Jiang M, Zheng X (2010) Interferon a-Inducible Protein 27 Computational Network Construction and Comparison between the Frontal Cortex of HIV Encephalitis (HIVE) and HIVE-Control Patients The Open Genomics Journal 3: 1-8.

26. Huang JX, Wang L, Jiang MH (2010) TNFRSF11B computational development network construction and analysis between frontal cortex of HIV encephalitis (HIVE) and HIVE-control patients. J Inflamm (Lond) 7: 50.

27. Sun L, Wang L, Jiang M, Huang J, Lin H (2011) Glycogen debranching enzyme 6 (AGL), enolase 1 (ENOSF1), ectonucleotide pyrophosphatase 2 (ENPP2 1) glutathione S-transferase 3 (GSTM3 3) and mannosidase (MAN2B2) metabolism computational network analysis between chimpanzee and human left cerebrum. Cell Biochem Biophys 61: 493-505.

28. Sun Y, Wang L, Jiang M, Huang J, Liu Z, et al. (2010) Secreted phosphoprotein 1 upstream invasive network construction and analysis of lung adenocarcinoma compared with human normal adjacent tissues by integrative biocomputation. Cell Biochem Biophys 56: 59-71.

29. Sun Y, Wang L, Lui L (2008) Integrative decomposition procedure and Kappa statistics set up ATF2 ion binding module in Malignant Pleural Mesothelioma (MPM). Frontiers of Electrical and Electronic Engineering in China 3: 381-387.

30. Wang L, Huang J, Jiang M (2011) CREB5 computational regulation network construction and analysis between frontal cortex of HIV encephalitis (HIVE) and HIVE-control patients. Cell Biochem Biophys 60: 199-207.

31. Wang L, Huang J, Jiang M (2010) RRM2 computational phosphoprotein network construction and analysis between no-tumor hepatitis/cirrhotic live tissues and human hepatocellular carcinoma (HCC). Cell Physiol Biochem 26: 303-310.

32. Wang L, Huang J, Jiang M, Lin H (2012) Signal Transducer and Activator of Transcription 2 (STAT2) Metabolism Coupling Postmitotic Outgrowth to Visual and Sound Perception Network in Human Left Cerebrum by Biocomputation. J Mol Neurosci.

33. Wang, L, Huang J, Jiang M, Sun L (2011) Survivin (BIRC5) cell cycle computational network in human no-tumor hepatitis/cirrhosis and hepatocellular carcinoma transformation. J Cell Biochem 112: 1286-1294. 
Citation: Huang J, Wang L, Jiang M, Lin H (2012) ALK Folic Acid Transport and Integrin Signal Induced-Angiogenesis Network in Human Hepatocellular Carcinoma (HCC) by Systems-Theoretical Analysis. Mol Biol 1:102. doi:10.4172/2168-9547.1000102

34. Wang L, Huang J, Jiang M, Sun L (2011) MYBPC1 computational phosphoprotein network construction and analysis between frontal cortex of HIV encephalitis (HIVE) and HIVE-control patients. Cell Mol Neurobiol 31: 233241.

35. Wang L, Huang J, Jiang M, Zheng X (2010) AFP computational secreted network construction and analysis between human hepatocellular carcinoma (HCC) and no-tumor hepatitis/cirrhotic liver tissues. Tumour Biol 31: 417-425.
36. Wang L, Sun Y, Jiang M, Zhang S, Woll S (2009) FOS proliferating network construction in early colorectal cancer (CRC) based on integrative significant function cluster and inferring analysis. Cancer Invest 27: 816-824.

37. Wang, L, Sun Y, Jiang M, Zheng X (2009) Integrative decomposition procedure and Kappa statistics for the distinguished single molecular network construction and analysis. J Biomed Biotechnol 2009: 726728. 